Journal of the Applied Mathematics, Statistics and Informatics (JAMSI), 8 (2012), No. 1

\title{
NEW INTEGRAL FORMULAS INVOLVING POLYNOMIALS AND I-FUNCTION
}

\author{
PRAVEEN AGARWAL AND SHILPI JAIN
}

\begin{abstract}
:
The aim of the present paper is to evaluate new finite integral formulas involving polynomials and $\overline{\mathrm{I}}$-function. The values of the formulas are obtained in terms of $\psi(\mathrm{z})$ (The logarithmic derivative of $\Gamma(\mathrm{z})$ ). These integral formulas are unified in nature and act as key formula from which we can obtain as their special cases. For the sake of illustration we record here some special cases of our main formulas which are also new and known. The formulas establish here are basic in nature and are likely to find useful applications in the field of science and engineering.
\end{abstract}

Additional Key Words and Phrases: İ-function, generalized polynomials, logarithmic derivative of $\Gamma(z)$

\section{INTRODUCTION}

The general class of polynomials $S_{n_{1}, \ldots, n_{r}}^{m_{1}, \ldots, m_{r}}[x]$ will be defined and represented as follows [1, p.185, Eqn. (7)]:

$$
S_{\mathrm{n}_{1}, \ldots, n_{r}}^{m_{1}, \ldots, m_{r}}[x]=\sum_{l_{1}=0}^{\left[\mathrm{n}_{1} / \mathrm{m}_{1}\right]} \cdots \sum_{l_{\mathrm{r}}=0}^{\left[\mathrm{n}_{\mathrm{r}} / \mathrm{m}_{\mathrm{r}}\right]} \prod_{i=1}^{r} \frac{\left(-\mathrm{n}_{\mathrm{i}}\right) \mathrm{m}_{\mathrm{i}} l_{\mathrm{i}}}{l_{\mathrm{i}} !} A_{n_{i}, l_{i}} \mathrm{x}^{l_{\mathrm{i}}}
$$

where $\mathrm{n}_{1}, \ldots, \mathrm{n}_{\mathrm{r}}=0,1,2, \ldots ; \mathrm{m}_{1}, \ldots, \mathrm{m}_{\mathrm{r}}$ are arbitrary positive integers, the coefficients $A_{n_{i}, l_{i}}$ $\left(n_{i}, l_{i} \geq 0\right)$ are arbitrary constants, real or complex. 


\section{P. AGARWAL, S. JAIN}

The $\bar{I}$-function will be defined and represented as follows [2,p.305, Eqn.6.5]

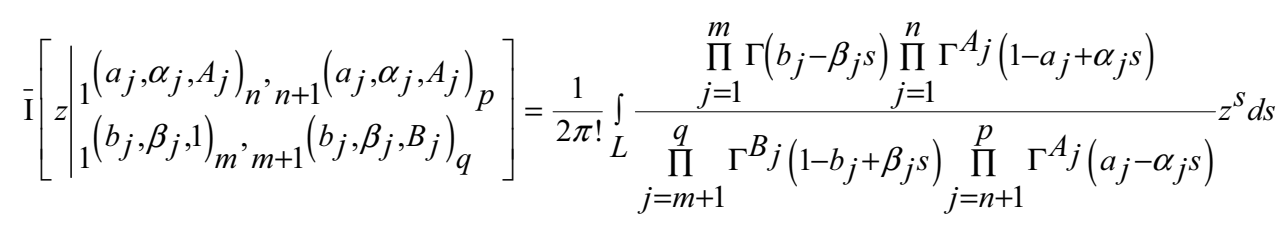

and

$$
A_{j}(j=1, \ldots, n), B_{j}(1, \ldots, m), m, n, p, q \quad \text { are } \quad \text { integers } \quad \text { satisfy }
$$

$0 \leq n \leq p, 1 \leq m \leq q$ and $\alpha_{j}, \beta_{j}$ are positive numbers and $a_{j}, b_{j}$ are complex numbers. In the sequel the I-function will be studied under the following conditions of existence:

(I) $\Omega>0,|\arg z|<\frac{\Omega \pi}{2}$

(II) $\Omega \geq 0,|\arg z| \leq \frac{\Omega \pi}{2}$ and $\operatorname{Re}(B+1)<0$

Where $\Omega=\sum_{j=1}^{n} A_{j} \alpha_{j}-\sum_{j=n+1}^{p} A_{j} \alpha_{j}+\sum_{j=1}^{m} \beta_{j}-\sum_{j=m+1}^{q} B_{j} \beta_{j}$,

And $B=\sum_{j=1}^{m} b_{j}+\sum_{j=m+1}^{q} b_{j}-\sum_{j=1}^{n} a_{j}-\sum_{j=n+1}^{p} a_{j}+\frac{1}{2}(p-q)$

By summing up the residues at the simple pole of the integrand in (2) we have by the application of Cauchy Residue theorem the following expansion for $\overline{\mathrm{I}}(z)$ after little simplification [2, p.305, Eqn. (6.8)]: 


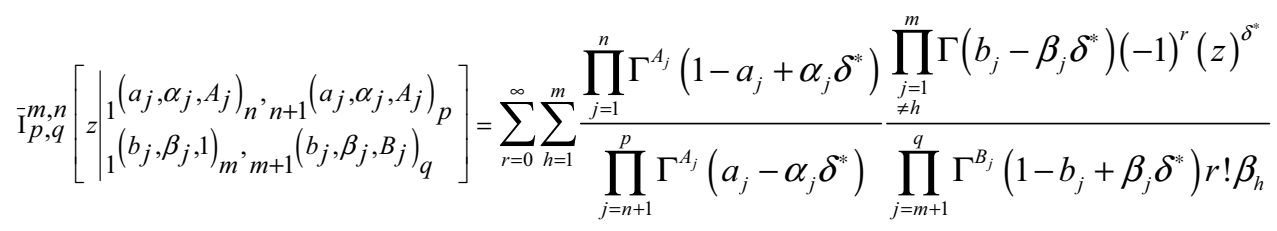

where $\delta^{*}=\frac{b_{h}+r}{\beta_{h}}$

Main Integrals: Let $\psi(z)$ denote the logarithmic derivative of gamma function $\Gamma(z)$ i.e.

$$
\psi(z)=\frac{\Gamma^{\prime}(z)}{\Gamma(z)}
$$

Also, let $\mathrm{E}(\mathrm{x})$ stands for $\left\{2+\lambda_{1}(1-x)+\lambda_{2}(1+x)\right\}$

And

$$
G(x)=\frac{(1-x)^{a-1}(1+x)^{b-1}}{\{E(x)\}^{a+b}} S_{\mathrm{n}_{1}, \ldots, n_{r}}^{m_{1}, \ldots, m_{r}}\left[c \prod_{i=1}^{r} \frac{(1-x)^{S_{i}}(1+x)^{t_{i}}}{\{E(x)\}^{s_{i}+t_{i}}}\right] \stackrel{-m, n}{\mathrm{I}} \mathrm{I}_{p, q}\left[y \frac{(1-x)^{\mu}(1+x)^{v}}{\{E(x)\}^{\mu+v}}\right]
$$

We have

$$
\int_{-1}^{1} G(x) \log \left(\frac{1-x}{E(x)}\right) \mathrm{dx}
$$


P. AGARWAL, S. JAIN

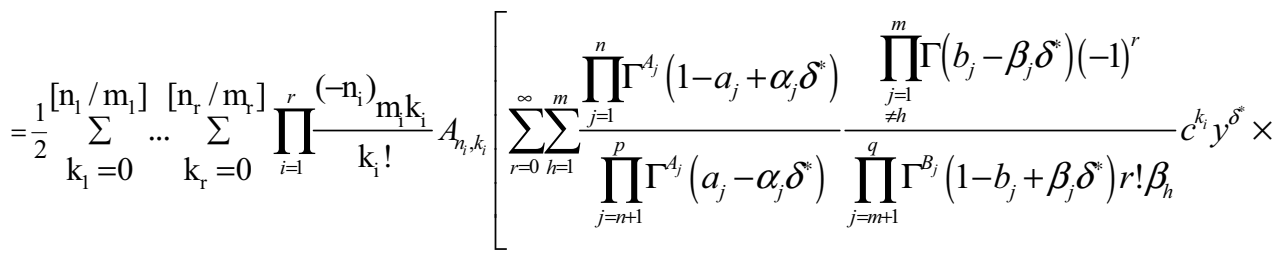

$\left\{\prod_{i=1}^{r}\left(1+\lambda_{1}\right)^{-s-\lambda_{i} k_{i}-\mu \delta^{*}}\left(1+\lambda_{2}\right)^{-t-\delta_{i} k_{i}-v \delta^{*}} B\left(a+s_{i} k_{i}+\mu \delta^{*}, b+t_{i} k_{i}+v \delta^{*}\right) \times\right.$

$\left.\left[-\log \left(1+\lambda_{1}\right)+\psi\left(a+s_{i} k_{i}+\mu \delta^{*}\right)-\psi\left(a+b+\left(s_{i}+t_{i}\right) k_{i}+(\mu+v) \delta^{*}\right)\right]\right]$

$\int_{-1}^{1} G(x) \log \left(\frac{1+x}{E(x)}\right) \mathrm{dx}$

$=\frac{1}{2} \sum_{\mathrm{k}_{1}=0}^{\left[\mathrm{n}_{1} / \mathrm{m}_{1}\right]} \ldots \sum_{\mathrm{k}_{\mathrm{r}}=0}^{\left[\mathrm{n}_{\mathrm{r}} / \mathrm{m}_{\mathrm{r}}\right]} \prod_{i=1}^{r} \frac{\left(-\mathrm{n}_{\mathrm{i}}\right)}{\mathrm{m}_{\mathrm{i}} !} \mathrm{k}_{\mathrm{i}} A_{n_{i}, k_{i}}\left[\sum_{r=0}^{\infty} \sum_{h=1}^{m} \frac{\prod_{j=1}^{n} \Gamma^{A_{j}}\left(1-a_{j}+\alpha_{j} \delta^{*}\right)}{\prod_{j=n+1}^{p} \Gamma^{A_{j}}\left(a_{j}-\alpha_{j} \delta^{*}\right)} \frac{\prod_{\substack{j=1 \\ \neq h}}^{m} \Gamma\left(b_{j}-\beta_{j} \delta^{*}\right)(-1)^{r}}{\prod_{j=m+1}^{q} \Gamma^{B_{j}}\left(1-b_{j}+\beta_{j} \delta^{*}\right) r ! \beta_{h}} c^{k_{i}} y^{\delta^{*}} \times\right.$

$\left\{\prod_{i=1}^{r}\left(1+\lambda_{1}\right)^{-a-s_{i} k_{i}-\mu \delta^{*}}\left(1+\lambda_{2}\right)^{-b-t_{i} k_{i}-v \delta^{*}} B\left(a+s_{i} k_{i}+\mu \delta^{*}, b+t_{i} k_{i}+v \delta^{*}\right) \times\right.$

$\left.\left.\left[-\log \left(1+\lambda_{2}\right)+\psi\left(b+t_{i} k_{i}+v \delta^{*}\right)-\psi\left(a+b+\left(s_{i}+t_{i}\right) k_{i}+(\mu+v) \delta^{*}\right)\right]\right]\right]$ 
NEW INTEGRAL FORMULAS INVOLVING POLYNOMIALS AND İ-FUNCTION

$\int_{-1}^{1} G(x) \log \left(\frac{1-x^{2}}{\{E(x)\}^{2}}\right) \mathrm{dx}$

$=\frac{1}{2} \sum_{\mathrm{k}_{1}=0}^{\left[\mathrm{n}_{1} / \mathrm{m}_{1}\right]} \cdots \sum_{\mathrm{k}_{\mathrm{r}}=0}^{\left[\mathrm{n}_{\mathrm{r}} / \mathrm{m}_{\mathrm{r}}\right]} \prod_{i=1}^{r} \frac{\left(-\mathrm{n}_{\mathrm{i}}\right)}{\mathrm{m}_{\mathrm{i}} \mathrm{k}_{\mathrm{i}}} A_{n_{i}, k_{i}}\left[\sum_{r=0}^{\infty} \sum_{h=1}^{m} \frac{\prod_{j=1}^{n} \Gamma^{A_{j}}\left(1-a_{j}+\alpha_{j} \delta^{*}\right)}{\prod_{j=n+1}^{p} \Gamma^{A_{j}}\left(a_{j}-\alpha_{j} \delta^{*}\right)} \frac{\prod_{\substack{j=1 \\ \neq h}}^{m} \Gamma\left(b_{j}-\beta_{j} \delta^{*}\right)(-1)^{r}}{\prod_{j=m+1}^{q} \Gamma^{\beta_{j}}\left(1-b_{j}+\beta_{j} \delta^{k}\right) r ! \beta_{h}} y^{\delta^{*}} \times\right.$

$\left\{\prod_{i=1}^{r}\left(1+\lambda_{1}\right)^{-a-s_{i} k_{i}-\mu \delta^{*}}\left(1+\lambda_{2}\right)^{-b-t_{i} k_{i}-v \delta^{*}} B\left(a+s_{i} k_{i}+\mu \delta^{*}, b+t_{i} k_{i}+v \delta^{*}\right) \times\right.$

$\left.\left.\left[-\log \left\{\left(1+\lambda_{1}\right)\left(1+\lambda_{2}\right)\right\}+\psi\left(a+s_{i} k_{i}+\mu \delta^{*}\right)+\psi\left(b+t_{i} k_{i}+v \delta^{*}\right)-2 \psi\left(a+b+\left(s_{i}+t_{i}\right) k_{i}+(\mu+v) \delta^{*}\right)\right]\right\}\right]$

$\int_{-1}^{1} G(x) \log \left(\frac{1-x}{1+x}\right) \mathrm{dx}$

$=\frac{1}{2} \sum_{\mathrm{k}_{1}=0}^{\left[\mathrm{n}_{1} / \mathrm{m}_{1}\right]} \cdots \sum_{\mathrm{k}_{\mathrm{r}}=0}^{\left[\mathrm{n}_{\mathrm{r}} / \mathrm{m}_{\mathrm{r}}\right]} \prod_{i=1}^{r} \frac{\left(-\mathrm{n}_{\mathrm{i}}\right)_{\mathrm{m}_{\mathrm{i}} \mathrm{k}_{\mathrm{i}}}}{\mathrm{k}_{\mathrm{i}} !} A_{n_{i}, k_{i}}\left[\sum_{r=0}^{\infty} \sum_{h=1}^{m} \frac{\prod_{j=1}^{n} \Gamma^{A_{j}}\left(1-a_{j}+\alpha_{j} \delta^{*}\right)}{\prod_{j=n+1}^{p} \Gamma^{A_{j}}\left(a_{j}-\alpha_{j} \delta^{*}\right)} \frac{\prod_{\substack{j=1 \\ \neq h}}^{m} \Gamma\left(b_{j}-\beta_{j} \delta^{*}\right)(-1)^{r}}{\prod_{j=m+1}^{q} \Gamma^{\beta_{j}}\left(1-b_{j}+\beta_{j} \delta^{*}\right) r ! \beta_{h}} c^{k_{i}} y^{\delta^{*}} \times\right.$

$\left\{\prod_{i=1}^{r}\left(1+\lambda_{1}\right)^{-a-s_{i} k_{i}-\mu \delta^{*}}\left(1+\lambda_{2}\right)^{-b-t_{i} k_{i}-v \delta^{*}} B\left(a+s_{i} k_{i}+\mu \delta^{*}, b+t_{i} k_{i}+v \delta^{*}\right) \times\right.$

$\left.\left.\left[-\log \left\{\frac{\left(1+\lambda_{1}\right)}{\left(1+\lambda_{2}\right)}\right\}+\psi\left(a+s_{i} k_{i}+\mu \delta^{*}\right)-\psi\left(b+t_{i} k_{i}+v \delta^{*}\right)\right]\right\}\right]$

The following interesting integral will be required to establish the results from (10) to (13):

$\int_{-1}^{1} \frac{(1-x)^{a-1}(1+x)^{b-1}}{\{E(x)\}^{a+b}} S_{\mathrm{n}_{1}, \ldots, n_{r}}^{m_{1}, \ldots, m_{r}}\left[c \prod_{i=1}^{r} \frac{(1-x)^{s_{i}}(1+x)^{t_{i}}}{\{E(x)\}^{s_{i}+t_{i}}}\right] \overline{\mathrm{I}}_{p, q}^{m, n}\left[y \frac{(1-x)^{\mu}(1+x)^{v}}{\{E(x)\}^{\mu+v}}\right] \mathrm{dx}$ 


$$
=\frac{1}{2} \sum_{\mathrm{k}_{1}=0}^{\left[\mathrm{n}_{1} / \mathrm{m}_{1}\right]} \cdots \sum_{\mathrm{k}_{\mathrm{r}}=0}^{\left[\mathrm{n}_{\mathrm{r}} / \mathrm{m}\right]} \prod_{i=1}^{r} \frac{\left(-\mathrm{n}_{\mathrm{i}}\right)_{\mathrm{m}} \mathrm{m}_{\mathrm{i}}}{\mathrm{k}_{\mathrm{i}} !} A_{n_{i}, k_{i}} c^{k_{i}}\left(1+\lambda_{\mathrm{r}}\right)^{-a-s_{i} \mathrm{k}_{\mathrm{i}}}\left(1+\lambda_{2}\right)^{-b-t_{i} \mathrm{k}_{\mathrm{i}}-m, m+2} \mathrm{I}_{p+2, q+1}\left[y\left(1+\lambda_{1}\right)^{-\mu}\left(1+\lambda_{2}\right)^{-v} \mid \begin{array}{l}
T_{1} \\
T_{2}
\end{array}\right]
$$

where $\left\{\begin{array}{l}T_{1} \equiv\left(1-a-s_{i} k_{i}, \mu, 1\right) ;\left(1-b-t_{i} k_{i}, v, 1\right) ;{ }_{1}\left(a_{j}, \alpha_{j}, A_{j}\right)_{n^{\prime}{ }_{n+1}}\left(a_{j}, \alpha_{j}, A_{j}\right)_{p} \\ T_{2} \equiv_{1}\left(b_{j}, \beta_{j}, 1\right)_{m^{\prime}{ }_{m+1}}\left(b_{j}, \beta_{j}, B_{j}\right)_{q} ;\left(1-a-b-\left(s_{i}+t_{i}\right) k_{i}, \mu+v, 1\right)\end{array}\right.$

$$
\begin{aligned}
& =\frac{1}{2} \sum_{\mathrm{k}_{1}=0}^{\left[\mathrm{n}_{1} / \mathrm{m}_{1}\right]} \sum_{\mathrm{k}_{\mathrm{r}}=0}^{\left[\mathrm{n}_{\mathrm{r}} / \mathrm{m}_{\mathrm{r}}\right]} \prod_{i=1}^{r} \frac{\left(-\mathrm{n}_{\mathrm{i}}\right)_{\mathrm{i}} \mathrm{k}_{\mathrm{i}}}{\mathrm{k}_{\mathrm{i}} !} A_{n_{i}, k_{i}}\left[\sum_{r=0}^{\infty} \sum_{h=1}^{m} \frac{\prod_{j=1}^{n} \Gamma^{A_{j}}\left(1-a_{j}+\alpha_{j} \delta^{*}\right)}{\prod_{j=n+1}^{p} \Gamma^{A_{j}}\left(a_{j}-\alpha_{j} \delta^{*}\right)} \frac{\prod_{\substack{j=1 \\
\neq h}}^{m} \Gamma\left(b_{j}-\beta_{j} \delta^{*}\right)(-1)^{r}}{\prod_{j=m+1}^{q} \Gamma^{\beta_{j}}\left(1-b_{j}+\beta_{j} \delta^{*}\right) r ! \beta_{h}} y^{\delta^{*} \times}\right. \\
& \left.\left\{\prod_{i=1}^{r}\left(1+\lambda_{1}\right)^{-a-s_{i} k_{i}-\mu \delta^{*}}\left(1+\lambda_{2}\right)^{-b-t_{i} k_{i}-v \delta^{*}} \frac{\Gamma\left(a+s_{i} k_{i}+\mu \delta^{*}\right) \Gamma\left(b+t_{i} k_{i}+v \delta^{*}\right)}{\Gamma\left(a+b+\left(s_{i}+t_{i}\right) k_{i}+(\mu+v) \delta^{*}\right)}\right\}\right]
\end{aligned}
$$

The above result will be converge under the conditions given by (3) and (4)

(I) $\operatorname{Re}(a)+\mu \min \left(\frac{b_{j}}{\beta_{j}}\right)>0$

(II) $\operatorname{Re}(b)+v \min \left(\frac{b_{j}}{\beta_{j}}\right)>0$

(III) All the parameters $a, b, s_{i}, t_{i}, k_{i}, \mu, v$ are positive. 
To evaluate the above integral we express $S_{\mathrm{n}_{1}, \ldots, n_{r}}^{m_{1}, \ldots, m_{r}}[x]$ in its series form with the help of

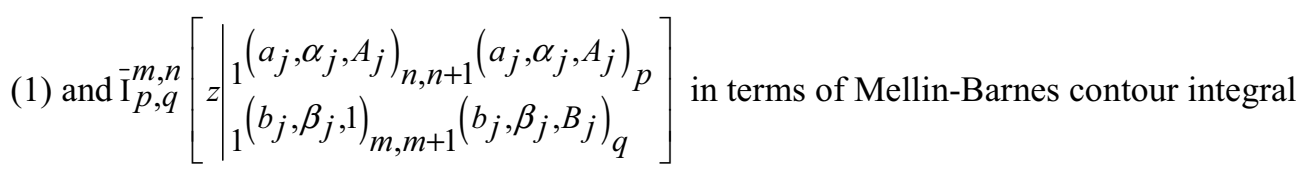
by (2), change the order of integration and summation and integrate the $\mathrm{x}$ - integral with the help of a known result recorded in the familiar text by Gradshteyan and Ryzhik [3, p.287]:

$$
\begin{aligned}
& \int_{a}^{b} \frac{(t-a)^{\alpha-1}(b-t)^{\beta-1}}{\{b-a+\lambda(t-a)+\mu(b-t)\}^{\alpha+\beta}} d t \\
& =\frac{(1+\lambda)^{-\alpha}(1+\mu)^{-\beta}}{b-a} \frac{\Gamma(\alpha) \Gamma(\beta)}{\Gamma(\alpha+\beta)}
\end{aligned}
$$

$a \neq b, \operatorname{Re}(\alpha)>0, \operatorname{Re}(\beta)>0, b-a+\lambda(t-a)+\mu(b-t) \neq 0$. Finally,

interpreting the $\xi$ contour integral in terms of the $\overline{\mathrm{I}}$-function, we arrive at the right hand side of (14).If we express the $\bar{I}$-function involved in the right hand side of (14) in series form with the help of (7), we easily arrive at (15).

Derivation of the Main Integrals: The result in (10) is established by taking the partial derivative of both sides of equation (15) with respect to a. (11) is similarly established by taking the partial derivative of equation (15) with respect to b.(12) and (13) are established the first adding the (10) and (11) then subtracting these.

Special Cases: On account of the most general nature of the $\bar{I}$-function and $S_{n_{1}, \ldots, n_{r}}^{m_{1}, \ldots, m_{r}}[x]$ occurring in our main integrals given by (10) to (13), a large number of integrals involving simpler functions of one variable can be easily obtained as their special cases. We however give here some special cases by way of illustration:

1) If we put $A_{j}=B_{j}=1$ in (10) $\bar{I}$-function reduces to the familiar Fox's H-function, then the result reduces to the following form:

$$
\int_{-1}^{1} \frac{(1-x)^{a-1}(1+x)^{b-1}}{\{E(x)\}^{a+b}} S_{\mathrm{n}_{1}, \ldots, n_{r}}^{m_{1}, \ldots, m_{r}}\left[c \prod_{i=1}^{r} \frac{(1-x)^{S_{i}}(1+x)^{t_{i}}}{\{E(x)\}^{S_{i}+t_{i}}}\right] \mathrm{H}_{p, q}^{m, n}\left[y \frac{(1-x)^{\mu}(1+x)^{v}}{\{E(x)\}^{\mu+v}}\right] \log \left(\frac{1+x}{E(x)}\right) \mathrm{dx}
$$




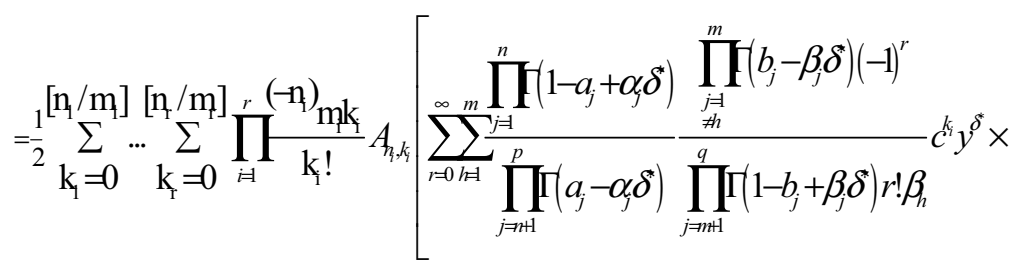

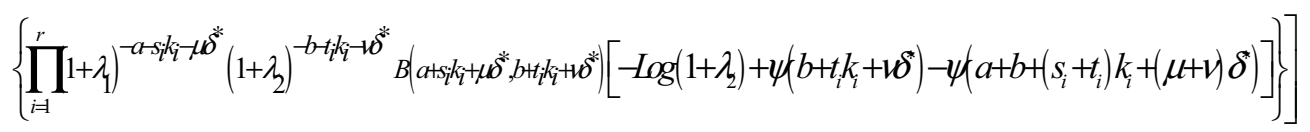

The above result is converge under the conditions given by (16) and (17) and

$$
\mathrm{A}>0,|\arg z|<\frac{\mathrm{A} \pi}{2} \text { where } \mathrm{A}=\sum_{j=1}^{n} \alpha_{j}+\sum_{j=1}^{m} \beta_{j}-\sum_{j=n+1}^{p} \alpha_{j}-\sum_{j=m+1}^{q} \beta_{j}
$$

2) If we take $\lambda_{1}=\lambda_{2}=0, c=1, m_{1}, \ldots, m_{r}=1, n_{1}, \ldots, n_{r}=n, s_{i}=1, t_{i}=0$ and $\mathrm{A}\left(\mathrm{n}_{1}, \mathrm{k}_{1}, \ldots, \mathrm{n}_{r}, \mathrm{k}_{r}\right)=\left(\begin{array}{l}n+\alpha \\ n\end{array}\right) \frac{(\alpha+\beta+n+1)_{k}}{(\alpha+1)_{k}}$, then the polynomial $S_{n}^{1}\left[\frac{1-x}{2}\right]$ occurring therein break up in to the Jacobi polynomials $P_{n}^{(\alpha, \beta)}(x)$ [4] and the integral (10) takes the following form after a little simplification:

$$
\begin{aligned}
& \int_{-1}^{1} \frac{(1-x)^{a-1}(1+x)^{b-1}}{\{2\}^{a+b} \log \left(\frac{1-x}{2}\right) \mathrm{P}_{n}^{(\alpha, \beta)}(x) \operatorname{I}_{p, q}^{m, n}\left[y \frac{(1-x)^{\mu}(1+x)^{v}}{\{2\}^{\mu+v}}\right] \mathrm{dx}} \\
& \quad=\frac{1}{2} \sum_{\mathrm{k}=0}^{n} \frac{(-n)_{l}(\alpha+\beta+n+1)_{l}}{l !(\alpha+1)_{l} 2^{l}}\left(\begin{array}{l}
n+\alpha \\
n
\end{array}\right)\left[\sum_{r=0}^{\infty} \sum_{h=1}^{m} \frac{\prod_{j=1}^{n} \Gamma^{A_{j}}\left(1-a_{j}+\alpha_{j} \delta^{*}\right)}{\prod_{j=n+1}^{p} \Gamma^{A_{j}}\left(a_{j}-\alpha_{j} \delta^{*}\right)} \frac{\prod_{\substack{j=1 \\
\neq h}}^{m} \Gamma\left(b_{j}-\beta_{j} \delta^{*}\right)(-1)^{r}}{\prod_{j=m+1}^{q} \Gamma^{B_{j}}\left(1-b_{j}+\beta_{j} \delta^{*}\right) r ! \beta_{h}} y^{\delta^{*}} \times\right. \\
& \left.\left\{\prod_{i=1}^{r} B\left(a+k_{i}+\mu \delta^{*}, b+v \delta^{*}\right)\left[\psi\left(a+k_{i}+\mu \delta^{*}\right)-\psi\left(a+b+k_{i}+(\mu+v) \delta^{*}\right)\right]\right\}\right]
\end{aligned}
$$

The above result is converge under the same condition as that of Eqn. (15) 
3) If we put $n=p=0, m=1, q=2, b_{1}=0, \beta_{1}=1, b_{m+1}=-\lambda^{\prime}, \beta_{m+1}=\mu^{\prime}, A_{j}=B_{J}=1 \overline{\mathrm{I}}$ function reduces to Wright's generalized Bessel function, i.e. $\overline{\mathrm{I}}_{0,2}^{1,0}\left[z \mid \begin{array}{l}(\ldots \ldots \ldots .) \\ (0,1,1),\left(-\lambda^{\prime}, \mu^{\prime}, 1\right)\end{array}\right]=J_{\lambda^{\prime}}^{\mu^{\prime}}(z)$ then result (18) reduces to

$\int_{-1}^{1} \frac{(1-x)^{a-1}(1+x)^{b-1}}{\{2\}^{a+b}} \log \left(\frac{1-x}{2}\right) \mathrm{P}_{n}^{(\alpha, \beta)}(x) J_{\lambda^{\prime}}^{\mu^{\prime}}\left[y \frac{(1-x)^{\mu}(1+x)^{\nu}}{\{2\}^{\mu+\nu}}\right] \mathrm{dx}$

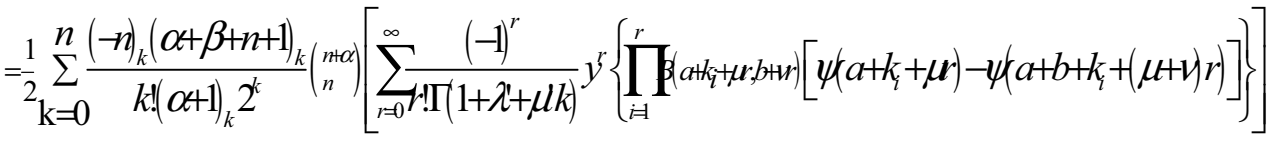

4) If we put $n=p, m=1, q=q+1, b_{1}=0, \beta_{1}=1, a_{j}=1-a_{j}, b_{j}=1-b_{j}, A_{j}=B_{J}=1$ in (18) I-function reduces to Wright's generalized Hypergeometric function, i.e. $\overline{\mathrm{I}}_{p, q+1}^{1, p}\left[z \mid \begin{array}{l}\left(1-a_{j}-\alpha_{j}\right)_{1, p} \\ (0,1),\left(1-b_{j}, \beta_{j}\right) 1, p\end{array}\right]={ }_{p} \psi_{q}\left[\begin{array}{l}\left(a_{j}, \alpha_{j}\right)_{1, p} ;-z \\ \left(b_{j}, \beta_{j}\right)_{1, p}\end{array}\right]$ then result reduces to $\int_{-1}^{1} \frac{(1-x)^{a-1}(1+x)^{b-1}}{\{2\}^{a+b}} \log \left(\frac{1-x}{2}\right) \mathrm{P}_{n}^{(\alpha, \beta)}(x){ }_{p} \psi_{q}\left[-y \frac{(1-x)^{\mu}(1+x)^{\nu}}{\{2\}^{\mu+\nu}}\right] \mathrm{dx}$

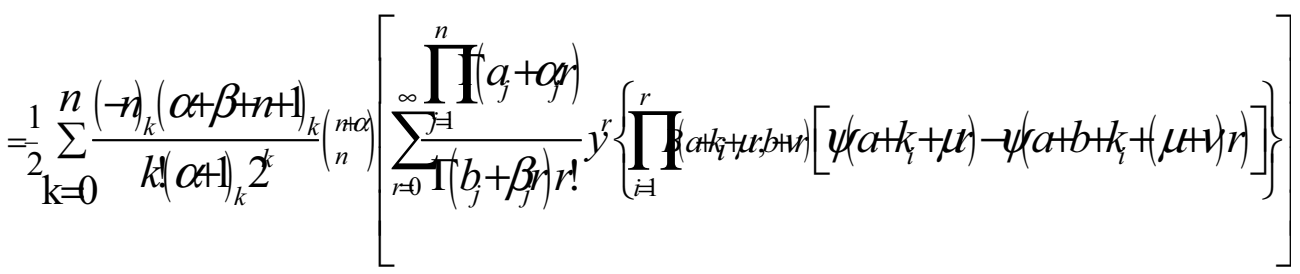

The above result is converge under the following conditions

I $\Delta>0,|\arg z|<\frac{\Delta \pi}{2}$ where $\Delta=\sum_{j=1}^{n} \alpha_{j}-\beta_{j}$ and equation (16) \& (17)

II The parameters $\alpha, \beta$ are positive. 


\section{Acknowledgements}

The authors are thankful to the Professor H. M. Srivastava (University of Victoria, Canada) for his kind help and suggestion in the preparation of this paper.

\section{References:}

[1] H.M.Srivastava, A multilinear generating function for the Konhauser sets of biorthogonal polynomials suggested by the Laguerre polynomials, Pacific J.Math.117, (1985), 183-191.

[2] Arjun K. Rathie, A New Generalization Of Generalized Hypergeometric Functions, Le Matematiche, Vol. LII (1997)-Fac.II, pp. 297-310

[3] I.S. Gradshteyan and Ryzhik, I.M.Tables of integrals, series and products, corrected and enlarged edition, New York: Academic Press, (1980).

[4] G.Szego, Orthogonal Polynomials.Amer.Math.Soc.Colloq.Publ.vol.23, 4th Ed. Amer. Math. Soc, Providence, Rhode Island, (1975).

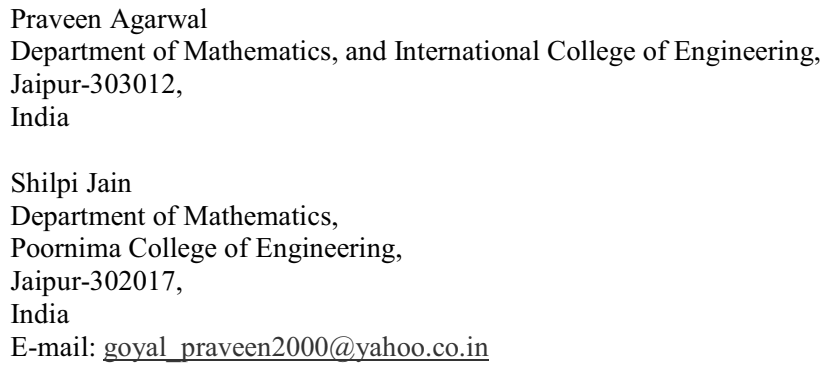

Received October 2011 